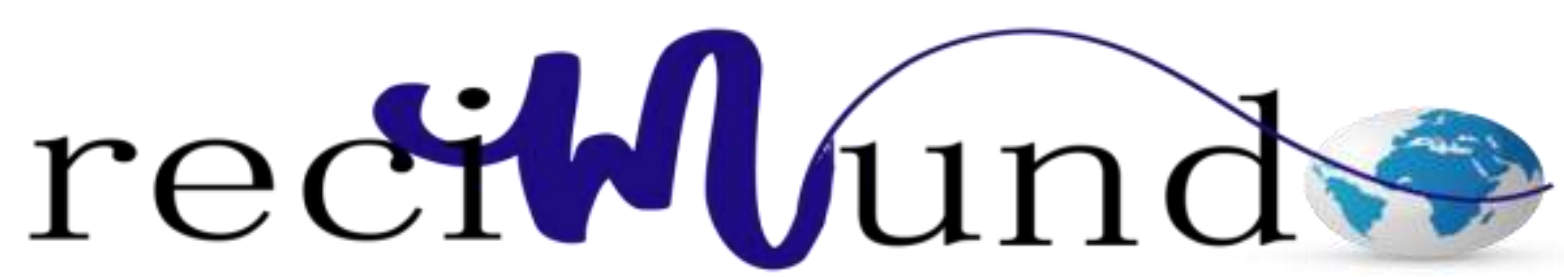

Revista Científica Mundo de la Investigación y el Conocimiento

\author{
Ronald Roossevelt Ramos Montiel ${ }^{\text {a; }}$ Gladys Eulalia Cabrera Cabrera b; Felipe \\ Rafael Calle Jara ${ }^{\text {c; }}$ Adriana Belén Romo Cardoso ${ }^{\mathrm{d}}$
}

Las tecnologías de la información y comunicación en los procesos de enseñanza en la educación superior

Information and communication technologies in teaching processes in higher education

Revista Científica Mundo de la Investigación y el Conocimiento. Vol. 3 núm.3. Esp., noviembre, ISSN: 2588-073X, 2019, pp. 706-724

DOI: $10.26820 /$ recimundo/3.(3.Esp).noviembre.2019.706-724

URL: http://recimundo.com/index.php/es/article/view/627

Código UNESCO: 1203 Ciencia de Los Ordenadores

Tipo de Investigación: Artículo de Revisión

(C) RECIMUNDO; Editorial Saberes del Conocimiento, 2019

Recibido: 15/09/2019

Aceptado: 23/10/2019

Publicado: 30/11/2019

Correspondencia: rramosm@ucacue.edu.ec

\footnotetext{
a. Diploma Superior en Odontología integral; Especialista en Ortodoncia; Odontólogo; Universidad Católica de Cuenca; Cuenca, Ecuador; rramosm@ucacue.edu.ec

b. Licenciada en ciencias de la educación Especialista en Docencia Universitaria Magíster en ciencias de la educación docente de pregrado de la Universidad Católica de Cuenca facultad de salud y bienestar carrera de Odontología; prof_gladysc@hotmail.com

c. Especialista en Implantología Buco Máxilo Facial; Odontólogo; Universidad Católica de Cuenca; Cuenca, Ecuador; fcallej@ucacue.edu.ec

d. Especialista en Odontología Pediátrica; Odontóloga; Investigadora Independiente; Cuenca, Ecuador; adrianaromoc@hotmail.com
} 


\section{Las tecnologías de la información y comunicación en los procesos de enseñanza en la educación superior}

Vol. 3, núm. 3 Esp., (2019)

Ronald Roossevelt Ramos Montiel; Gladys Eulalia Cabrera Cabrera; Calle Jara Felipe Rafael;

Adriana Belén Romo Cardoso

\section{RESUMEN}

Uno de los problemas más habituales que ha tenido la sociedad, en toda su historia, es el poder comunicarse de manera eficaz y efectiva. En la era globalizada este problema se diluye gracias a las diferentes herramientas que pueda otorgar las tecnologías de la información y comunicación. Las universidades no escapan de ser los baluartes en la utilización de estos medios y más si estos brindan la oportunidad acercar la información en cualquier espacio y en tiempo real. Tanto, los docentes como los estudiantes tienen el compromiso de poder generar el conocimiento apoyados con estas herramientas digitales, tantas plataformas educativas como de las redes sociales, no sin antes recibir el apoyo de las políticas emanadas de las instituciones y de los organismos del estado que les facilite el uso de estas tecnologías. Por lo cual, esta investigación tiene como objetivo general analizar la influencia de las tecnologías de la información y comunicación en los procesos de enseñanza en la educación superior. La metodología utilizada se basa en investigaciones de carácter documental y bibliográfica. Los resultados se basaron en el análisis de los métodos pedagógicos utilizados en las instituciones de educación superior, la influencia de la era globalizada a través de las plataformas educativas y de las redes sociales como factores catalizadores en la generación del conocimiento y en el compromiso que puedan tener los protagonistas universitarios en la utilización de estas herramientas. Como conclusión se tiene que el apoyo con las diferentes herramientas hace que el conocimiento se construya de una manera más socializada dentro de las instituciones de educación superior, la cual permite formar un nuevo paradigma basado en el constructivismo y el conectivismo, es decir, se elabora el conocimiento entre todos a través de la conexión que se tenga con el mundo exterior por medio de plataformas digitales.

Palabras Clave: Comunicación; Tecnología; Plataformas digitales; Redes sociales. 


\section{Las tecnologías de la información y comunicación en los procesos de enseñanza en la educación superior}

Vol. 3, núm. 3 Esp., (2019)

Ronald Roossevelt Ramos Montiel; Gladys Eulalia Cabrera Cabrera; Calle Jara Felipe Rafael; Adriana Belén Romo Cardoso

\section{ABSTRACT}

One of the most common problems that society has had, throughout its history, is being able to communicate effectively and effectively. In the globalized era, this problem is diluted thanks to the different tools that information and communication technologies can provide. Universities do not escape being the bastions in the use of these means and more if they provide the opportunity to bring information in any space and in real time. Both teachers and students have the commitment to generate knowledge supported by these digital tools, both educational platforms and social networks, but not before receiving the support of the policies emanating from the institutions and agencies of the state that facilitate the use of these technologies. Therefore, this research has as a general objective to analyze the influence of information and communication technologies in teaching processes in higher education. The methodology used is based on documentary and bibliographic research. The results were based on the analysis of the pedagogical methods used in higher education institutions, the influence of the globalized era through educational platforms and social networks as catalytic factors in the generation of knowledge and in the commitment that the University protagonists in the use of these tools. In conclusion, the support with the different tools means that knowledge is constructed in a more socialized way within higher education institutions, which allows to form a new paradigm based on constructivism and connectivism, that is, it is elaborated knowledge among all through the connection that is had with the outside world through digital platforms.

Keywords: Communication; Technology; Digital platforms; Social networks. 


\section{Las tecnologías de la información y comunicación en los procesos de enseñanza en la educación superior}

Vol. 3, núm. 3 Esp., (2019)

Ronald Roossevelt Ramos Montiel; Gladys Eulalia Cabrera Cabrera; Calle Jara Felipe Rafael; Adriana Belén Romo Cardoso

\section{Introducción.}

La enseñanza en la educación superior ha estado basada en la formación a través de la teoría del conductivismo, donde una persona posee los conocimientos y esta lo transfiere a los demás dentro de un protocolo universitario comprendido en diferentes formas pedagógicas y utilizando las herramientas más comunes en la evaluación de la comprensión del conocimiento. Es decir, la formación del conocimiento es a través del diálogo directo entre el docente y el estudiante, donde la experiencia y conocimiento del primero prevalece sobre el segundo.

Desde la invención del internet, estas teorías pedagógicas han cambiado permitiendo un dinamismo superior en las aulas de clase. ya el conocimiento no es solo bajo la experiencia del educador sino bajo la experiencia de todo aquel que hace vida dentro del recinto universitario. Esta herramienta tecnológica ha permitido poder crear y fomentar una germinación de conocimiento que ha crecido de manera exponencial. Este conocimiento está al alcance de todos.

El internet forma parte del instrumento de las tecnologías de la información y comunicas (TIC). Estas TIC no solo han tomado protagonismo en los recintos universitarios sino también en cualquier arista productiva u organizacional. La información tiene infinidades de vehículos para ser transportadas por lo que se genera una comunicación más efectiva entre los miembros de una sociedad.

Por supuesto, esta nueva ola de la enseñanza a quebrado ciertos paradigmas educacionales. Todavía estos ejerciendo una gran fuerza de oposición debido a que fomentan el conformismo y la zona de confort. El debate constante que se generan en las aulas de clase es 


\section{Las tecnologías de la información y comunicación en los procesos de enseñanza en la educación superior}

Vol. 3, núm. 3 Esp., (2019)

Ronald Roossevelt Ramos Montiel; Gladys Eulalia Cabrera Cabrera; Calle Jara Felipe Rafael; Adriana Belén Romo Cardoso

producto de nuevas formas de buscar el conocimiento, pero sobre todo aplicación de nuevas herramientas pedagógicas que implican un gasto de energía y tiempo.

Pero este debate genera diálogos que pueden ir fomentan y generando los saberes que se deben conocer sobre cualquier punto. El dialogo siempre ha sido parte fundamental en la comunicación de la sociedad. El dialogo ha permitido poder evolucionar y por ende conocer las soluciones a los problemas que han aquejado al hombre a lo largo de la historia. El diálogo pertenece a la naturaleza del ser humano, en cuanto ser de comunicación, así como sella el acto de aprender, que nunca es individual, aunque tenga una dimensión individual(Carneiro, 2009).

Ahora, si existe una realidad compleja entre las universidades de primer mundo y las universidades del tercer mundo. Para poder cumplir con los objetivos de una educación moderna basada en la globalización se deben cumplir ciertas condiciones que van más allá de las instituciones educativas. Estas condiciones son de carácter político y socioeconómico. Esto depende d ellos gobernantes de turno y su visión de poder entender cuan necesario es el cambio en los paradigmas pedagógicos en la educación superior.

"Todas las organizaciones educativas y en particular, las universidades latinoamericanas y caribeñas, requieren redefinir su visión integral o diferenciar sus funciones académicas: docencia, investigación y extensión sobre un nuevo paradigma como eje transformador que le permita, a través de la creación y difusión del conocimiento, lograr la correspondencia entre lo que la sociedad demanda por una parte y la coherencia interna que debe reinar en las Universidades por la otra. Un sistema educativo que tome como relevantes programas que promueven la integración efectiva 


\section{Las tecnologías de la información y comunicación en los procesos de enseñanza en la educación superior}

Vol. 3, núm. 3 Esp., (2019)

Ronald Roossevelt Ramos Montiel; Gladys Eulalia Cabrera Cabrera; Calle Jara Felipe Rafael; Adriana Belén Romo Cardoso

entre las comunidades, el entorno social, con las áreas interdisciplinarias y transdisciplinarias del currículum, por ejemplo, pedagógica, psicológica y social; propiciando programas de desarrollo integral, con acento en la acción social comunitaria”. (Acosta \& Genyelbert, 2013, pág. 84).

Por lo que este cambio deben hacerlo todos aquellos que están involucrados e interesados por la calidad de la generación de conocimiento, sabiendo que este permitirá obtener una mejor educación y a su vez un mejor desarrollo social. Solo esto sucede si el educador tiene las herramientas suficientes para poder transmitir sus ideas y que tenga la capacidad de aceptar las ideas de los demás en un franco dialogo en pro de los saberes y las soluciones a los problemas de la sociedad. Empoderar a quien quiera compartir lo que sabe para encontrarse con aquellos que quieren aprender de él y por lo cual Dar la oportunidad para presentar al público general las propias ideas (Reig-Hernández, 2010).

Por lo cual, esta investigación tiene como objetivo general analizar la influencia de las tecnologías de la información y comunicación en los procesos de enseñanza en la educación superior. La metodología utilizada se basa en investigaciones de carácter documental y bibliográfica.

\section{Metodología.}

Esta investigación se basó en el detalle y análisis de los métodos pedagógicos utilizados en las instituciones de educación superior, las herramientas utilizadas como medios de comunicación, la influencia de la era globalizada en especial las tecnologías de la información y 


\section{Las tecnologías de la información y comunicación en los procesos de enseñanza en la educación superior}

Vol. 3, núm. 3 Esp., (2019)

Ronald Roossevelt Ramos Montiel; Gladys Eulalia Cabrera Cabrera; Calle Jara Felipe Rafael; Adriana Belén Romo Cardoso

comunicación como factores catalizadores en la generación del conocimiento, el desarrollo de plataformas educativas y de las redes sociales en el apoyo que pueden brindar en las aulas de clases y en el compromiso que puedan tener los protagonistas universitarios en la utilización de estas herramientas. Todo esto con el fin de poder determinar la influencia de las TIC en el proceso de enseñanza en la educación superior. Para esto se debe aplicar una metodología de carácter documental y bibliográfica a través de herramientas como textos, documentos y artículos científicos publicados disponibles en la web.

\section{Resultados.}

Poder producir un cambio significativo dentro de los estándares de educación universitaria no depende solamente de las políticas internas de las instituciones y mucho menos de las políticas nacionales sobre el avance tecnológico de la educación en el país. esto depende en gran medida en la actitud del educador y del educando. Las TIC no son más que herramientas importantes e interesantes que pueden ser utilizadas para poder incrementar la generación del conocimiento. Si no hay el interés de aquellos que tiene el deber de hacerlo de nada valdrá el tener los mejores laboratorios y talleres con la más alta tecnología. Es decir, la pedagogía y las ganas de aprender se transforman en un carácter netamente subjetivo.

"Las TIC, como tales, no aportan gran cosa ni al proceso de enseñanzaaprendizaje ni en términos de calidad ni de eficiencia. Es decir, podríamos afirmar que las TIC no pueden impactar positivamente la calidad del aprendizaje de los estudiantes si en la filosofía del diseño de enseñanza-aprendizaje no se aprovechan plenamente las posibilidades que ofrecen dichas tecnologías”. (Rodríguez-Izquierdo, 2011, pág. 19). 


\section{Las tecnologías de la información y comunicación en los procesos de enseñanza en la educación superior}

Vol. 3, núm. 3 Esp., (2019)

Ronald Roossevelt Ramos Montiel; Gladys Eulalia Cabrera Cabrera; Calle Jara Felipe Rafael; Adriana Belén Romo Cardoso

Este cambio acelerado que se han venido suscitando en esta era globaliza a permitido que la transformación empiece a ocurrir de manera lenta. Es la decisión de los actores del conocimiento que pueden permitir que las TIC tengan gran auge en el desarrollo de las ideas y soluciones. Los estudiantes y profesores se ven abocados a cambios trascendentales en su forma de enseñar y aprender, por lo que los cambios siempre son graduales en el mundo educativo real, muy a pesar de que las tecnologías cambien de manera desaforada (Quintero, 2009).

De aquí nace la comparación entre dos modelos educativos que actualmente pueden existir en las universidades, el modelo tradicional conductivista o el modelo apoyado en las TIC denominado modelo constructivista-conectivista. Las diferencias se pueden detallar en la Tabla 1.

Tabla 1. Diferencias entre los modelos que pueden existir en las instituciones de educación superior.

\begin{tabular}{|c|c|c|}
\hline Modelo Educativo & Trad & las TIC \\
\hline Rol del docente & $\begin{array}{l}\text { Es el centro de enseñanza, } \\
\text { informa } \\
\text { terminados. }\end{array}$ & $\begin{array}{l}\text { Sale del rol clásico y forma parte } \\
\text { de la clase con sus estudiantes. Ya } \\
\text { no es el protagonista. }\end{array}$ \\
\hline Rol d & $\begin{array}{l}\text { Poco margen para pensar. } \\
\text { Se le exige memorización. } \\
\text { Tiene un rol pasivo }\end{array}$ & $\begin{array}{l}\text { Adquiere mayor autonomía y } \\
\text { responsabilidad en el proceso de } \\
\text { aprendizaje. }\end{array}$ \\
\hline \multirow[t]{2}{*}{$\begin{array}{l}\text { Características de } \\
\text { la clase }\end{array}$} & $\begin{array}{l}\text { Exposición de la clase. } \\
\text { Poca cabida a experiencias } \\
\text { vivenciales. } \\
\text { Los contenidos se ofrecen } \\
\text { desvinculados de la realidad. }\end{array}$ & $\begin{array}{l}\text { Requiere un docente actualizado } \\
\text { con acceso oportuno a las TIC. } \\
\text { Potencia las experiencias de } \\
\text { aprendizaje, gracias a las } \\
\text { herramientas disponibles. }\end{array}$ \\
\hline & $\begin{array}{l}\text { Se evalúan resultados a nivel } \\
\text { reproductivo. }\end{array}$ & $\begin{array}{l}\text { Facilita el seguimiento del } \\
\text { aprendizaje. }\end{array}$ \\
\hline
\end{tabular}




\section{Las tecnologías de la información y comunicación en los procesos de enseñanza en la educación superior}

Vol. 3, núm. 3 Esp., (2019)

Ronald Roossevelt Ramos Montiel; Gladys Eulalia Cabrera Cabrera; Calle Jara Felipe Rafael; Adriana Belén Romo Cardoso

Fuente: (Albán, Arguello, Albán, \& Soledispa, 2019).

Esta oposición al cambio, por parte de aquellos que tienen el protagonismo de hacerlo, tanto docentes como estudiantes, no ha evitado que las universidades empiecen a trabajar en función de este desarrollo tecnológico. Poco a poco han entendido que la solución a muchos de los problemas que acontecen a la sociedad está en la forma en cómo se aborda y en la manifestación de las soluciones que se pueden dar. Estas instituciones empezaron a cambiar su perspectiva y han empezado a involucrarse con las TIC a través del uso de plataformas digitales. De manera tal que no se puede decir que haya impactado en tanto cambio socio institucional con la suficiente fuerza en la educación superior, manteniéndose las plataformas educativas basadas en TIC en un plano muy rezagado en comparación con la enseñanza tradicional (Rojas, Sorroza, Villacis, Caraguay, \& Sánchez, 2018).

El crecimiento de estas tecnologías ha permitido que la información sea abrumadora. Es allí donde los estudiantes y docentes deben saber discernir entre cual es aquella que de verdad tienen valor significativo sobre el tema de interés y cuáles no. Esto es debido a que el uso del internet ha servido para que se manejen informaciones no confirmadas por lo que a veces se entrelazan con las investigaciones con valor y peso científico.

"Se debe ser bien crítico al momento de evaluar la información que se toma como fuente de conocimiento, ya que esta masificación de información contenida en las redes también permite la inclusión de material de baja calidad; por lo cual se recomienda, encontrar lugares y buscadores que contengan información de fuentes confiables, de esta manera siempre impartir conocimiento de calidad y actualizado para 


\section{Las tecnologías de la información y comunicación en los procesos de enseñanza en la educación superior}

Vol. 3, núm. 3 Esp., (2019)

Ronald Roossevelt Ramos Montiel; Gladys Eulalia Cabrera Cabrera; Calle Jara Felipe Rafael; Adriana Belén Romo Cardoso

mantener los estándares de educación establecidos en cualquier institución docente”. (Iñiguez, Gómez, Lao, \& Ballesteros, 2018).

Es aquí donde los docentes tienen un papel importante en el desarrollo del conocimiento porque no solo es saber diferenciar sino por qué y para que se utilizan los métodos y técnicas de diferentes procesos. Los profesores tienen un papel importante en el desarrollo de nuevas ideas y para ellos su compromiso es fundamental por lo que las herramientas que utilice ayudaran al estudiante a comprender mucho mejor los conocimientos y con ello poder clasificar la información que pueda conseguir en la red de internet. Por lo que, la universidad tiene este gran desafío. Debe acercar la cultura del aprendizaje informal de los jóvenes a la formación donde su orientación y guía para un uso apropiado de la comunicación y las fuentes de información académicas es vital (Escofet Roig, García Gonzalez, \& Gros Salvat, 2011).

Con esto, la calidad de la educación en estas instituciones aumenta considerablemente. Existen mecanismos que permiten poder determinar la calidad de la educación. Esta calidad está determinada por:

"Ser operacional, es decir poder traducirse en elementos fácilmente manejables dentro de una guía, modelo o procedimiento de evaluación; Que abarque de una u otra forma las funciones sustantivas de la universidad; Que lleve implícito el concepto de apreciación o evaluación; y Que esté ligado a la pertinencia social”. (Cabrera, 2005, pág. 5). 


\section{Las tecnologías de la información y comunicación en los procesos de enseñanza en la educación superior}

Vol. 3, núm. 3 Esp., (2019)

Ronald Roossevelt Ramos Montiel; Gladys Eulalia Cabrera Cabrera; Calle Jara Felipe Rafael; Adriana Belén Romo Cardoso

Esta calidad de la educación universitaria está enmarcada en muchos factores. En

Ecuador predomina los recursos humanos y los recursos físicos que corresponden a la tecnología utilizada para el desarrollo del conocimiento. Estos permiten dar cuenta de las características de los actores y las principales dinámicas del sistema, mejorar la evaluación de los programas e instrumentos promoviendo una adecuación inteligente de los mismos a los objetivos planteados(Milia, 2014).

Esto aunado a las políticas nacionales ecuatorianas enmarcadas en la tercera ola de la transformación de la educación superior han permitido un crecimiento en la calidad de sus productos, es decir profesionales con fuerte carácter social y sentido de pertenencia.

"Recuperar lo público del bien educación superior implica ciertos lineamientos programáticos que deben llevarse a cabo, entre los cuales destacan: a) la descorporativización del campo; b) la democratización de la educación superior y del conocimiento; c) la construcción de un sistema que genere conocimiento, en el marco de una autonomía universitaria responsable y pertinente con la sociedad; d) la revalorización de la carrera del docente e investigador, eliminando la ausencia de reglas y la precarización laboral; e) la endogeneización regional del sistema de educación superior en América Latina y en el sur global; f) la convergencia cualitativa de excelencia de las instituciones de educación superior (IES), eliminando circuitos diferenciados de calidad; y, g) la construcción de una episteme emancipadora, a través de un nuevo régimen académico". (Gallegos, 2013, págs. 7-8). 


\section{Las tecnologías de la información y comunicación en los procesos de enseñanza en la educación superior}

Vol. 3, núm. 3 Esp., (2019)

Ronald Roossevelt Ramos Montiel; Gladys Eulalia Cabrera Cabrera; Calle Jara Felipe Rafael; Adriana Belén Romo Cardoso

Por otra parte, la educación universitaria ecuatoriana debe dar el paso para la educación 2.0 es decir formar sus clases a través de la educación a distancia con la ayuda de las plataformas digitales, tanto comerciales o sociales como las de finalidad educativa. Los docentes deben apoyarse en esta metodología, combinando sus procesos tradicionales con la ayuda tecnológica que ofrece estos medios. En Ecuador, tan solo el 27\% de estas han incorporado plataformas educativas, entre las causas principales de esa mínima implementación tenemos: falta de conocimiento de los directivos, recursos tecnológicos y económicos insuficientes, o la escasa difusión (Tamayo, 2012). En la Figura 1 se detalla las plataformas virtuales educativas que se pueden utilizar de herramientas para el desarrollo del conocimiento.

Figura 1. Plataformas virtuales educativas que se pueden utilizar de herramientas para desarrollo del conocimiento

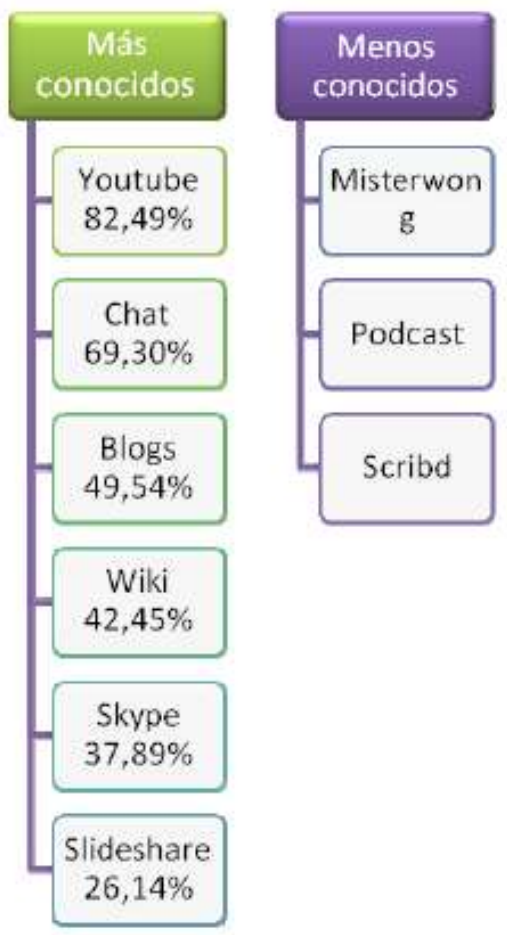




\section{Las tecnologías de la información y comunicación en los procesos de enseñanza en la educación superior}

Vol. 3, núm. 3 Esp., (2019)

Ronald Roossevelt Ramos Montiel; Gladys Eulalia Cabrera Cabrera; Calle Jara Felipe Rafael; Adriana Belén Romo Cardoso

Fuente: (Tamayo, 2012).

Con el apoyo de estas plataformas digitales más la plataforma de educación digital, como Moodle, se puede entrelazar un sistema de gestión de aprendizaje (LMS) más efectiva. Por lo que, mediante su adopción y el cumplimiento de estándares, se transforman en unidades de innovación que han permitido fomentar la educación superior con modalidades presencial, con soporte de aula virtual, semipresencial, virtual y a distancia (Guanoluisa \& Herrera, 2018). En la Tabla 2 se detalla, los LMS utilizadas en las universidades ecuatorianas.

Tabla 2. Los LMS utilizadas en las universidades ecuatorianas

\begin{tabular}{ll}
\hline Universidad & LMS \\
\hline Escuela Politécnica Nacional & MOODLE \\
\hline Universidad San Francisco De Quito & DESIRE2LEARN, D2L \\
\hline Escuela Superior Politécnica del Litoral & CANVAS \\
\hline Universidad Espíritu Santo (UEES) & BLACKBOARD \\
\hline Facultad Latinoamericana de Ciencias Sociales Sede Ecuador (FLACSO) & BLACKBOARD \\
\hline Universidad Andina Simón Bolívar & MOODLE \\
\hline Universidad de Fuerzas Armadas ESPE & MOODLE \\
\hline Universidad de Cuenca & MOODLE \\
\hline Escuela Politécnica del Chimborazo & MOODLE \\
\hline Pontificia Universidad Católica del Ecuador & MOODLE \\
\hline Universidad de las Américas (UDLA) & MOODLE \\
\hline
\end{tabular}

Fuente: (Guanoluisa \& Herrera, 2018).

Una de las plataformas más dinámicas y de gran aceptación en toda la sociedad como vehículo de comunicación son las redes sociales. Esta interactividad es importante y se debe 


\section{Las tecnologías de la información y comunicación en los procesos de enseñanza en la educación superior}

Vol. 3, núm. 3 Esp., (2019)

Ronald Roossevelt Ramos Montiel; Gladys Eulalia Cabrera Cabrera; Calle Jara Felipe Rafael; Adriana Belén Romo Cardoso

destacar como herramienta pedagógica por su carácter amigable y flexible. Este aprovechamiento debe hacer surgir ideas paras el desarrollo de cualquier contenido académico.

"Los distintos estilos de educación se han vuelto importantes al proporcionar a los estudiantes una participación activa, comunicarse en un ambiente flexible, compartir información universal, personalizar la educación y el entorno para sí mismos y aprender de forma independiente, sin importar lugar y tiempo. De acuerdo con estas expectativas, es de entender que la eficacia se incrementará cuando las redes sociales, una de las tecnologías emergentes, comiencen a ser utilizadas activa y razonablemente en el campo educativo". (Pazmiño, Mendieta, Murrieta, \& Soto, 2019, págs. 891-892).

Esta flexibilidad, eficiencia de transferir la comunicación y el dominio de las masas sobre estas plataformas convierten a las redes sociales como medios de información especiales e importantes para el desarrollo del conocimiento por parte de las instituciones de educación superior ecuatorianas. Las redes sociales se constituyen en herramientas de generación y retroalimentación de información, en donde el modelo de comunicación resulta eficiente e innovador, aunque todavía es necesario continuar masificando su uso y buscar sus utilidades para incorporarlos a los procesos de aprendizaje (Téllez \& Trujillo, 2018).

El apoyo del uso de las redes sociales es tan grande que muchos de los ecuatorianos que tienen teléfonos inteligentes tienen instalados alguna de estas plataformas, como se detalla en la Tabla 3.Las preferencias, que tienen los estudiantes, acerca de las formas de comunicarse con otros; priorizan a las redes sociales y a las redes para compartir: videos, presentaciones visuales, 


\section{Las tecnologías de la información y comunicación en los procesos de enseñanza en la educación superior}

Vol. 3, núm. 3 Esp., (2019)

Ronald Roossevelt Ramos Montiel; Gladys Eulalia Cabrera Cabrera; Calle Jara Felipe Rafael;

Adriana Belén Romo Cardoso

imágenes y documentos; así como también, los gestores de correo electrónico(Humanante Ramos, García-Peñalvo, Conde González, \& Velasco-Silva, 2015).

Tabla 3. Población con celular inteligente y redes sociales a nivel nacional

\begin{tabular}{ccccc}
\hline Período & $\begin{array}{c}\text { Población } \\
\text { Total }\end{array}$ & $\begin{array}{c}\text { Población } \\
\text { que tiene } \\
\text { teléfono } \\
\text { celular } \\
\text { activado }\end{array}$ & $\begin{array}{c}\text { Población que } \\
\text { tiene teléfono } \\
\text { celular } \\
\text { SMARTPHONE }\end{array}$ & $\begin{array}{c}\text { Población que } \\
\text { en su teléfono } \\
\text { smartphone } \\
\text { utiliza redes } \\
\text { sociales }\end{array}$ \\
\hline dic-11 & 14.478 .129 & 6.209 .858 & 522.640 & 365.427 \\
dic-12 & 14.682 .556 & 6.859 .938 & 839.705 & 641.914 \\
dic-13 & 15.872 .755 & 7.453 .781 & 1.261 .944 & 1.081 .620 \\
dic-14 & 16.148 .648 & 7.820 .597 & 1.928 .108 & 1.722 .159 \\
dic-15 & 16.404 .531 & 8.174 .520 & 3.084 .886 & 2.807 .282 \\
dic-16 & 16.714 .929 & 8.482 .236 & 4.484 .087 & 4.224 .984 \\
dic-17 & 16.961 .926 & 8.987 .750 & 5.716 .770 & 5.412 .575 \\
\hline
\end{tabular}

Fuente: (ENEMDU, 2018).

Estas herramientas permiten poder acercar la información en el aula de clase y poder transformarla en los conocimientos necesarios para poder solventar algún problema. Es de destacar que sin el interés del docente estas herramientas son utilizadas de manera banal y sin eficiencia por lo que el facilitador se convierte en pieza fundamental en el desarrollo de los contenidos a través de estas plataformas virtuales.

"Por lo tanto, la relación que tienen los docentes con estas competencias digitales es muy factible, siempre y cuando se tenga la convicción al cambio por parte del facilitador y 


\section{Las tecnologías de la información y comunicación en los procesos de enseñanza en la educación superior}

Vol. 3, núm. 3 Esp., (2019)

Ronald Roossevelt Ramos Montiel; Gladys Eulalia Cabrera Cabrera; Calle Jara Felipe Rafael; Adriana Belén Romo Cardoso

que también cuente con el apoyo en equipo e infraestructura por parte de los institutos de educación superior”. (Vera, Suárez, Suarez, \& Cruz, 2019, pág. 970).

\section{Conclusiones.}

Una de las características que tiene la educación universitaria es el desarrollo de los conocimientos. Dichos análisis provienen del debate socializado que pueda ocurrir en las aulas de clases. Estos diálogos son producto de la construcción de ideas que puedan tener para solventar alguna inquietud que pueda tener la sociedad a la cual se rodea. Esto convierte a las universidades en un factor social por lo que estas deben tener pertenencia en el colectivo y un profundo valor hacia los verdaderos problemas que puedan aquejar a la sociedad. Las universidades deben convertirse en verdaderos focos de ciencia que puedan solventar las necesidades de las poblaciones y no solo en recintos de debate interno.

Es por ello, que las universidades deben abrirse a nuevos horizontes dejando atrás los viejos paradigmas y establecer nuevas prioridades dentro de sus misiones y visiones de una mejor sociedad. Poder establecer este contacto con los problemas más críticos es urgente. El mundo globalizado ha llegado para entrelazar todas las aristas que pueda tener la sociedad con el fin de que puedan solventar los verdaderos problemas que los aquejan. Dentro de esta globalización están las tecnologías de la información y comunicación.

Estas herramientas han permitido construir todo el conocimiento posible gracias a la generación de información que de ella emana. El apoyo con las diferentes herramientas hace que el conocimiento se construya de una manera más socializada dentro de las instituciones de 


\section{Las tecnologías de la información y comunicación en los procesos de enseñanza en la educación superior}

Vol. 3, núm. 3 Esp., (2019)

Ronald Roossevelt Ramos Montiel; Gladys Eulalia Cabrera Cabrera; Calle Jara Felipe Rafael; Adriana Belén Romo Cardoso

educación superior. Se forma un nuevo paradigma basado en el constructivismo y el conectivismo, es decir, se elabora el conocimiento entre todos a través de la conexión que se tenga con el mundo exterior por medio de plataformas digitales.

Por supuesto, que el poder contar con estas plataformas requiere de apoyo desde la universidad como de los entes gubernamentales. Debe existir un compromiso de políticas que permitan la inversión en espacios destinados a la conectividad y de una adaptación de estos medios por parte del recurso humano que lo va a manejar. De nada sirve tener las plataformas sino hay un compromiso por parte del facilitador. Estas políticas deben permitir acercar el conocimiento global para que sea transformado en soluciones que permiten obtener las respuestas necesarios e importantes que requiera la sociedad.

Ahora, el compromiso radica en los docentes y estudiantes que tengan la posibilidad de enfrentarse a este dilema y hacer real el apoyo de estos medios como vehículos de información en tiempo real con el fin de poder generar el conocimiento. La utilización de diversas plataformas educativas aunado a 1 apoyo que se puedan dar con las redes sociales es vital en la generación de ideas y planteamientos acordes a los contenidos académicos y en función de la pertenencia social que se deba tener. Los docentes deben apoyarse en el uso de las redes sociales como factor catalizador en la conexión del conocimiento debido a lo flexible y amigable que es su manejo.

Por lo tanto, es un compromiso de las universidades, del estado, pero sobre todo de los protagonistas del conocimiento estar vinculados a los problemas reales de la sociedad, tomando en consideración los avances que ha tenido las tecnologías de la información y comunicación que 


\section{Las tecnologías de la información y comunicación en los procesos de enseñanza en la educación superior}

Vol. 3, núm. 3 Esp., (2019)

Ronald Roossevelt Ramos Montiel; Gladys Eulalia Cabrera Cabrera; Calle Jara Felipe Rafael; Adriana Belén Romo Cardoso

han tenido en los últimos años. Este compromiso es en la búsqueda de una mejor calidad de vida en los miembros de la sociedad.

\section{Bibliografía.}

Acosta, O., \& Genyelbert, E. (2013). Globalización e internacionalización de la educación superior un enfoque epistemológico. Omnia,, 19(1), 75-85.

Albán, G. P., Arguello, A. E., Albán, C. S., \& Soledispa, E. E. (2019). Las Tecnologías de la Información y la Comunicación en la educación universitaria. RECIAMUC, 3(3), 409422.

Cabrera, V. A. (2005). El concepto calidad en la educación universitaria: clave para el logro de la competitividad institucional. Revista iberoamericana de Educación, 36(12), 1-7.

Carneiro, R. (2009). Las TIC y los nuevos paradigmas educativos: la transformación de la escuela en una sociedad que se transforma. En R. Carneiro, J. C. Toscano, \& T. Díaz, Los desafíos de las TIC para el cambio educativo (págs. 15-28). Madrid, España: Fundación Santillana.

ENEMDU. (2018). Tecnologías de la Información y Comunicación. Quito-Ecuador: Instituto Nacional de Estadística y Censos.

Escofet Roig, A., García Gonzalez, I., \& Gros Salvat, B. (2011). Las nuevas culturas de aprendizaje y su incidencia en la educación superior. Revista mexicana de investigación educativa, 16(51), 1177-1195.

Gallegos, R. (2013). Tercera ola de transformación de la educación superior en Ecuador. QuitoEcuador: Secretaría Nacional de Educación Superior, Ciencia, Tecnología e Innovación.

Guanoluisa, D. M., \& Herrera, L. A. (2018). Estudio de innovación tecnológica aplicada en procesos formativos con sistemas de gestión de aprendizaje (LMS) en la educación universitaria del Ecuador. Revista Ibérica de Sistemas e Tecnologias de Informação, E15, 256-270.

Humanante Ramos, P. R., García-Peñalvo, F. J., Conde González, M. Á., \& Velasco-Silva, D. P. (2015). Diagnóstico del uso de los dispositivos electrónicos y de las herramientas web 2.0 desde un enfoque PLE en un grupo de estudiantes de ingeniería. Madrid, España: III Congreso Internacional sobre Aprendizaje, Innovación y Competitividad (CINAIC 2015).

Iñiguez, E. L., Gómez, M. E., Lao, J. M., \& Ballesteros, H. F. (2018). Las TIC y su incidencia en la producción de información estratégica educacional. RECIAMUC, 2(2), 432-445. 


\section{Las tecnologías de la información y comunicación en los procesos de enseñanza en la educación superior}

Vol. 3, núm. 3 Esp., (2019)

Ronald Roossevelt Ramos Montiel; Gladys Eulalia Cabrera Cabrera; Calle Jara Felipe Rafael;

Adriana Belén Romo Cardoso

Milia, M. F. (2014). Marco de Políticas Públicas de Ciencia, Tecnología y Educación Superior en el Ecuador. Nuevos horizontes: dinámicas y condicionamientos para una Investigación Universitaria de cara a la Sociedad. Buenos Aires, Argentina: Congreso Iberoamericano de Ciencia, Tecnología, Innovación y Educación.

Pazmiño, E. R., Mendieta, M. A., Murrieta, G. V., \& Soto, M. A. (2019). Importancia y uso de las redes sociales en la educación. RECIMUNDO, 3(2), 582-593.

Quintero, L. J. (2009). Las universidades apostando por las TIC: modelos y paradojas de cambio institucional. EDUTEC. Revista Electrónica de Tecnología Educativa(28), 1-14.

Reig-Hernández, D. (2010). El futuro de la educación superior, algunas claves. REIRE. Revista d'Innovació i Recerca en Educació, 3(2), 98-113.

Rodríguez-Izquierdo, R. M. (2011). Repensar la relación entre las TIC y la enseñanza universitaria: problemas y soluciones. Profesorado. Revista de currículum y formación del profesorado, 15(1), 9-22.

Rojas, N. A., Sorroza, J. P., Villacis, J. E., Caraguay, W. A., \& Sánchez, M. V. (2018). Las TIC y la resistencia al cambio en la educación superior. RECIMUNDO: Revista Científica de la Investigación y el Conocimiento, 2(2), 477-495.

Tamayo, C. F. (2012). Plataformas virtuales como recurso para la enseñanza en la universidad: análisis, evaluación y propuesta de integración de moodle con herramientas de la web 2.0. Madrid, España: Trabajo de Grado - Universidad Complutende se Madrid, Facultad de Educación .

Téllez, M. N., \& Trujillo, E. P. (2018). Las redes sociales en el contexto académico universitario. Desafios al docente.Etic@net, 18(1),83-101.

Vera, C. F., Suárez, L. X., Suarez, M. N., \& Cruz, A. R. (2019). Competencias digitales en docentes: desafío de la educación superior. RECIAMUC, 3(3), 946-974.

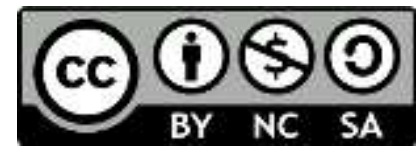

\section{RECONOCIMIENTO-NOCOMERCIAL-COMPARTIRIGUAL}

CC BY-NC-SA

ESTA LICENCIA PERMITE A OTROS ENTREMEZCLAR, AJUSTAR Y CONSTRUIR A PARTIR DE SU OBRA CON FINES NO COMERCIALES, SIEMPRE Y CUANDO LE RECONOZCAN LA AUTORÍA Y SUS NUEVAS CREACIONES ESTÉN BAJO UNA LICENCIA CON LOS MISMOS TÉRMINOS. 